\title{
TENSORS DETERMINED BY A HYPERSURFACE IN RIEMANN SDACE*
}

\author{
BY \\ HARRY LEVY \\ INTRODUCTION
}

Bianchi $\nmid$ has generalized the study of a surface in euclidean 3-space by considering a hypersurface, $V_{n}$, immersed in an arbitrary enveloping space of one more dimension. Associated with such a hypersurface there are two differential quadratic forms, which in this paper we denote by $g_{\alpha \beta} d u^{\alpha} d u^{\beta}$ and $\Omega_{\alpha \beta} d u^{\alpha} d u^{\beta}$, where the $u^{\prime}$ s $(\alpha, \beta=1,2, \cdots, n)$ are coördinates in $V_{n}$. These forms, known as the first and second fundamental forms of the hypersurface respectively, are defined precisely as are the two fundamental forms of a surface in ordinary 3-space; the first in the case of a positive definite form gives the square of the element of the arc,

$$
d s^{2}=g_{\alpha \beta} d u^{\alpha} d u^{\beta}
$$

while the second may be obtained by considering the variation of the first order of the fundamental tensor of the space as one passes from the given hypersurface to a nearby hypersurface geodesically parallel to the given one. The variations of higher order, not considered by Bianchi, yield additional tensors which are intimately connected with the study of the given hypersurface and of the hypersurfaces geodesically parallel to the given one. These tensors can be found by writing the linear element of the enveloping space in the form

$$
\varphi=e\left(d u^{0}\right)^{2}+c_{\alpha \beta} d u^{\alpha} d u^{\beta},
$$

where the curves of parameter $u^{0}$ are geodesics orthogonal to $V_{n}$ and the parameter $u^{0}$ is the arc of these geodesics measured from $V_{n}$. The coefficients in the expression of $c_{\alpha \beta}$ as a power series in $u^{0}$ are the required sequence of tensors,

$$
c_{\alpha \beta}=g_{\alpha \beta}-2 \Omega_{\alpha \beta} u^{0}+\cdots \cdot
$$

The hypersurfaces $u^{0}=$ constant are geodesically parallel and the fundamental tensor of any one of them is given by this power series with $u^{0}$

\footnotetext{
* Presented to the Society, February 28, 1925; received by the editors in February, 1925.

$\dagger$ Bianchi, Lezioni di Geometria Differenziale, 2d edition, vol. I, or 3d edition, vol. II. Reference may also be made to other works that have recently appeared, Der Ricci-Kalkül, by J. A. Schouten; Lezioni di Calcolo Differenziale Assoluto, by T. Levi-Civita; Vorlesungen über Differentialgeometrie, II, by W. Blaschke; Riemannian Geometry, by L. P. Eisenhart, Princeton University Press.
} 
replaced by the particular value of the constant. Hence these remaining terms are essential, in particular, in the study of geodesically parallel hypersurfaces.

We devote the first part of the paper to a brief capitulation of known results, and to the proof of two theorems in general tensor analysis which we need in the later development. In the second part we obtain the general form of this sequence of tensors, expressing it in intrinsic form in terms of known functions of the space and hypersurface. In the last part of the paper we discuss some subcases, proving several theorems of geometric interest.

The author wishes to express his appreciation of the valuable suggestions and helpful criticism given him by Professor Eisenhart.

\section{PART I}

1. We consider a Riemann space of $n+1$ dimensions, denoted for brevity by $V_{n+1}$; the fundamental form of this space*

$$
\varphi=a_{r s} d x^{r} d x^{s},
$$

is assumed to have a non-vanishing determinant,

$$
a=\left|a_{r s}\right| \neq 0 \text {. }
$$

We denote by $a^{r s}$ the cofactor of $a_{r s}$ in the determinant $a$ divided by $a$ itself. We may then write

$$
a_{r s} a^{s t}=\delta_{r}^{t} \quad(r, t=0,1,2, \cdots, n),
$$

where $\delta_{r}^{t}$ is a Kronecker delta, that is,

$$
\left.\delta_{r}^{t}=\begin{array}{l}
0, r \neq t \\
1, r=t
\end{array}\right\} \quad(r, t=0,1, \cdots, n) .
$$

Let $[r s, t]$ and $\left\{r_{r}{ }_{s}\right\}$ be the Christoffel symbols of the first and second kinds respectively, so that

$$
[r s, t]=\frac{1}{2}\left(\frac{\partial a_{r t}}{\partial x^{s}}+\frac{\partial a_{s t}}{\partial x^{r}}-\frac{\partial a_{r s}}{\partial x^{t}}\right)
$$

and

$$
\left\{\begin{array}{c}
p \\
r s
\end{array}\right\}=a^{p t}[r s, t], \quad[r s, t]=a_{p t}\left\{\begin{array}{c}
p \\
r s
\end{array}\right\}
$$

\footnotetext{
* As usual, the repetition of an index, once as subscript and once as superscript, indicates a summation on that index over all values from 0 to $n$. However, compare the remarks following equations (4.3).
} 
We need also the Riemann symbols of the fourth order; denote those of the first kind by $R_{p r s t}$ and those of the second kind by $R_{r s t}^{p}$, so that

$$
\begin{aligned}
& R_{p r s t}=\frac{\partial}{\partial x^{t}}[p s, r]-\frac{\partial}{\partial x^{s}}[p t, r]+\left\{\begin{array}{c}
k \\
p t
\end{array}\right\}[r s, k]-\left\{\begin{array}{c}
k \\
p s
\end{array}\right\}[r t, k] \\
& R_{p s t}^{r}=\frac{\partial}{\partial x^{t}}\left\{\begin{array}{c}
r \\
p s
\end{array}\right\}-\frac{\partial}{\partial x^{s}}\left\{\begin{array}{c}
r \\
p t
\end{array}\right\}+\left\{\begin{array}{c}
k \\
p s
\end{array}\right\}\left\{\begin{array}{c}
r \\
k t
\end{array}\right\}-\left\{\begin{array}{c}
k \\
p t
\end{array}\right\}\left\{\begin{array}{c}
r \\
k s
\end{array}\right\}
\end{aligned}
$$

and

$$
R_{r s t}^{p}=a^{p q} R_{r q s t}, \quad R_{p r s t}=a_{r q} R_{p s t}^{q} .
$$

These relations may be written in a number of other ways because of (1.5), (1.6) and (1.9).* The properties of the $R$ 's, namely that

$$
\begin{gathered}
R_{r s t}^{p}=-R_{r t s}^{p}, \\
R_{r s t}^{p}+R_{s t r}^{p}+R_{t r s}^{p}=0, \\
R_{p r s t}=-R_{p r t s}=R_{r p t s}=R_{t s r p},
\end{gathered}
$$

follow quite readily from the definitions. The reader is referred to Bianchi for further details.

Let us denote the covariant derivative of $R_{\text {prst }}$ with respect to $x^{\varepsilon}$ and the form (1.1) by $R_{p r s t, e}$ and similarly let $R_{r s t, e}^{p}$ be the covariant derivative of $R_{r s t}^{p}$. Since the covariant derivatives of $a_{i j}$ and $a^{i j}$ vanish, $\dagger$ it follows from (1.9) that

$$
R_{r s t, e}^{p}=a^{p q} R_{r q s t, e}, \quad R_{p r s t, e}=a_{r q} R_{p s t, e}^{q} .
$$

Let us recall also the identity of Bianchif

$$
R_{r s t, u}^{p}+R_{r t u, s}^{p}+R_{r u s, t}^{p}=0 .
$$

Successive covariant differentiation of $R_{r s t, u}^{p}$ and $R_{\text {prst,u }}$ gives us a sequence of tensors which we denote by $R_{r s t, u}^{p} \ldots v$ and $R_{p r s t, u} \ldots v$. We find that these $R$ 's satisfy the analogues of (1.13) and the equations obtained through the differentiation of (1.10), (1.11), (1.12) and (1.14).

* Bianchi, 2d edition, loc. cit., pp. 72, 73.

$\dagger$ Ricci and Levi-Civita, Méthodes de calcul différential absolu, Ma th e ma tis ch e Annalen, vol. 54 (1901), p. 138.

$\ddagger$ Bianchi, loc. cit., p. 351. Also Veblen, Normal coördinates for the geometry of paths, Proceedings of the National Academy of Sciences, vol. 8 (1922), p. 197. 
2. Consider a point function $f\left(x^{0}, x^{1}, \cdots, x^{n}\right)$ evaluated along a curve $C$. If we write

$$
\lambda^{r}=\frac{d x^{r}}{d s} \quad(r=0,1,2, \cdots, n),
$$

the $\lambda$ 's are the components of the vector tangent to the curve, $s$ being its arc; $*$ the derivative of $f$ along $C$ is given by

$$
\frac{\partial f}{\partial s}=f_{r} \lambda r
$$

where $f_{r}$ is the derivative $\partial f / \partial x^{r}$. The second derivative, $\partial^{2} f / \partial s^{2}$, can be obtained by differentiating the right hand side of (2.2) covariantly with respect to $x^{t}$ and the form (1.1), multiplying by $\lambda$, and summing on $t$. We obtain

$$
\frac{\partial^{2} f}{\partial s^{2}}=f_{r} \lambda^{2} \lambda^{\imath}+f_{r} \lambda^{r},{ }_{i} \lambda^{t}
$$

where $f_{r t}$ and $\lambda_{r, t}$ are the covariant derivatives of $f_{r}$ and $\lambda^{r}$ respectively.

If $C$ is a geodesic, and if we denote its arc by $u^{0}$, we must have $\dagger$

$$
\frac{d^{2} x^{r}}{\left(d u^{0}\right)^{2}}+\left\{\begin{array}{c}
r \\
i j
\end{array}\right\} \frac{d x^{i}}{d u^{0}} \frac{d x^{i}}{d u^{0}}=0 \quad(r=0,1,2, \cdots, n) .
$$

This may be written in the equivalent form $\ddagger$

$$
\lambda^{r}, \lambda^{i}=0,
$$

where $\lambda^{r}, i$ is the covariant derivative of $\lambda^{r}$ with respect to $x^{i}$ and the form (1.1). In equations (2.3) the second term drops out by virtue of equations (2.5), so that we have

$$
\frac{\partial^{2} f}{\left(\partial u^{0}\right)^{2}}=f_{r s} \lambda^{r} \lambda^{s} .
$$

By induction it follows that

$$
\frac{\partial^{m} f}{\left(\partial u^{0}\right)^{m}}=f_{r_{1} r_{2} \ldots r_{m}} \lambda^{r_{1}} \lambda^{r_{2}}-\lambda^{r_{m}} \quad(m=1,2, \cdots) .
$$

* If $C$ is not a mull curve, that is, if, for $C, a_{i j} d x^{i} d x^{j} \neq 0$, the arc is defined, $d s^{2}=\left|a_{i j} d x^{i} d x^{2}\right|$. If $C$ is null, $s$ must be interpreted as a parameter along the curve. Cf. Eisenhart, loc. cit.

† Bianchi, 2d edition, loc. cit., p. 334, or any of the other works on this subject.

I Cf. Levi-Civita, loc. cit., p. 291, or any of the similar works. 
Hence we have the following theorem:

The mth directional derivative of any function $f$ along a geodesic is obtained by taking the inner product of the mth covariant derivative of $f$ with $\lambda^{r^{2}} \lambda^{r_{2}} \cdots \lambda^{r^{-m}}$, where $\lambda^{r}$ is the vector tangent to the geodesic.

In particular, it follows that, if $-x^{i}$ is taken for the function $f$ we must have

$$
-\frac{d^{m} x^{i}}{\left(d u^{0}\right)^{m}}=\Gamma_{r_{1} \ldots r_{m}}^{i} \frac{d x^{r_{1}}}{d u^{0}} \cdots \frac{d x^{r_{m}}}{d u^{0}} \quad(i=0,1,2, \cdots, n),
$$

where the functions $\Gamma_{n_{1}}^{1} \ldots r_{m}$ are formed from $-x^{i}$ by the formal process of covariant differentiation,

$$
\begin{aligned}
& \left.\Gamma_{r_{1}}^{i}=\begin{array}{r}
0, r_{1}=i \\
-1, r_{1} \neq i
\end{array}\right\}, \\
& \Gamma_{r s}^{i}=\frac{\partial \Gamma_{r}^{i}}{\partial x^{s}}-\Gamma_{i}^{i}\left\{\begin{array}{c}
j \\
r s
\end{array}\right\} \equiv\left\{\begin{array}{c}
i \\
r s
\end{array}\right\}, \\
& \Gamma_{r s t}^{i}=\frac{\delta \Gamma_{r s}^{i}}{\delta x^{i}}-\Gamma_{j s}^{i}\left\{\begin{array}{c}
j \\
r t
\end{array}\right\}-\Gamma_{r j}^{i}\left\{\begin{array}{c}
j \\
s t
\end{array}\right\},
\end{aligned}
$$

Hence that solution of equations (2.4) which has initial values $y^{i}$ and $\xi^{i}$,

must be*

$$
\left.x^{i}\right|_{u^{0}=0}=y^{i},\left.\quad \frac{d x^{i}}{d u^{0}}\right|_{u^{0}=0}=\xi^{i},
$$

$$
x^{i}=y^{i}+\xi^{i} u^{0}-\frac{1}{2 !}\left\{\begin{array}{c}
i \\
r s
\end{array}\right\} \xi^{r} \xi^{s}\left(u^{0}\right)^{2}-\frac{1}{3 !} \Gamma_{r s t}^{i} \xi^{r} \xi^{s}\left(u_{0}\right)^{3}
$$

where $\left\{r^{i}\right\}$ and the $\Gamma$ 's are evaluated for the point $y^{i}$.

3. We consider an arbitrary tensor $T_{i j} \ldots k$ expanded in a power series, for simplicity, in one variable,

$$
\begin{aligned}
T_{i j \ldots k}=T_{i j \cdots k}^{(0)}+T_{i j \cdots k}^{(1)}\left(x^{0}\right)+ & T_{i j \cdots k}^{(2)}\left(x^{0}\right)^{2}+\cdots \\
& (i, j, k=0,1,2, \cdots, n) .
\end{aligned}
$$

If we make the transformation of coördinates defined by the equations

$$
\begin{aligned}
x^{0} & =\bar{x}^{0}, \\
x^{i} & =x^{i}\left(\bar{x}^{1}, \bar{x}^{2}, \cdots, \bar{x}^{n}\right) \quad(i=1,2, \cdots, n),
\end{aligned}
$$

* Cf. Veblen, loc. cit., p. 192. 
the components $\bar{T}_{i j} \ldots k$ in the new coördinate system undergo the usual tensor transformations, which in this case, and for a covariant tensor, reduce to

$$
\begin{aligned}
\vec{T}_{\alpha \beta \cdots \gamma}=\sum_{\lambda, \mu, \nu=1}^{n} T_{\lambda \mu} \ldots, \frac{\partial x^{\lambda}}{\partial \bar{x}^{\alpha}} \frac{\partial x^{\mu}}{\partial \bar{x}^{\beta}} & \cdots \frac{\partial x^{\nu}}{\partial \bar{x}^{\gamma}} \\
& (\alpha, \beta, \gamma=1,2, \cdots, n) .
\end{aligned}
$$

Since $x^{0}$ appears only in $T$ and not in the derivatives, it follows by differentiation of (3.3) with respect to $x^{0}$ that

$$
\bar{T}_{\alpha \beta}^{(m)} \cdots \gamma=\sum_{\lambda, \mu, \nu}^{1 \cdots n} T_{\lambda \mu \ldots \nu} \frac{\partial x^{\lambda}}{\partial \bar{x}^{\alpha}} \frac{\partial x^{\mu}}{\partial \bar{x}^{\beta}} \cdots \frac{\partial x^{\nu}}{\partial \bar{x}^{\gamma}},
$$

that is, that $T_{\lambda \mu \ldots,}^{(m)}$ are the components of a covariant tensor in $x^{0}=0$. The similar result for a mixed or contravariant tensor or invariant obviously is valid. If the expansion (3.1) is in a series of several variables, the result holds for the variety of the remaining variables. Putting this in the form of a theorem, we have

If $T_{i j} \ldots{ }_{k}$ and $\bar{T}_{i j} \ldots{ }_{k}$ are the components of a tensor in the coördinate systems $x$ and $\bar{x}$ respectively, and if these coordinates are related by equations

$$
\begin{array}{lrl}
x^{\alpha} & =\bar{x}^{\alpha} & (\alpha=0,1,2, \cdots, p-1), \\
x^{\mu}=x^{\mu}\left(\bar{x}^{p}, \bar{x}^{p+1}, \cdots, \bar{x}^{n}\right) & (\mu=p, p+1, \cdots, n),
\end{array}
$$

then the coefficients in the expansion of $T_{\lambda \mu} \ldots$ in a power series in $x^{0}, x^{1}$, ..., $x^{p-1}$ are the components of a tensor in the subspace $x^{\alpha}=$ constant $(\alpha=0,1, \cdots, p-1)$, and the components of this tensor in the coördinates $\bar{x}^{p}, \ldots, \bar{x}^{n}$ are the coefficients in the expansion of $\bar{T}_{\lambda \mu} \ldots$, in a power series in $x^{0}, x^{1}, \cdots, x^{p-1}$.

\section{PART II}

4. We consider a hypersurface of $n$ dimensions denoted by $V_{n}$ immersed in $V_{n+1}$ with the quadratic form (1.1). Such a spread is defined by the equations

$$
x^{i}=f^{i}\left(u^{1}, u^{2}, \cdots, u^{n}\right) \quad(i=0,1,2, \cdots, n),
$$

where the jacobian matrix

$$
J=\left\|\frac{\partial f^{i}}{\partial u^{\alpha}}\right\| \quad\left(\begin{array}{rr}
i=0,1,2, \cdots, n \\
\alpha=1,2, \cdots, n
\end{array}\right)
$$

is of rank $n$. The fundamental form of $V_{n}$ is given by

$$
\varphi^{\prime}=g_{\alpha \beta} d u^{\alpha} d u^{\beta}
$$


where

$$
g_{\alpha \beta}=a_{i j} \frac{\partial f^{i}}{\partial u^{\alpha}} \frac{\partial f^{j}}{\partial u^{\beta}} \quad(\alpha, \beta=1,2, \cdots, n) .
$$

In equations (4.3), $\alpha$ and $\beta$ are summed from 1 to $n$, and throughout the remainder of this paper Greek letters will be reserved for such summations, while Latin letters as $i$ and $j$ in (4.4) indicate a summation from 0 to $n$.

When the form (1.1) is positive definite, (4.3) will be likewise; but when no assumption is made concerning the definiteness of (1.1), it is necessary to assume explicitly that the functions $f^{i}$ are such that the discriminant $g$ of the form (4.3),

$$
g=\left|g_{\alpha \beta}\right|
$$

is different from zero.

As usual, $g^{\alpha \beta}$ denotes the cofactor of $g_{\alpha \beta}$ in $g$ divided by $g$ itself, so that

$$
g_{\alpha \beta} g^{\beta \gamma}=\delta_{\alpha}^{\gamma} \quad(\alpha, \gamma=1,2, \cdots, n),
$$

where $\delta_{\alpha}^{\gamma}$ is defined by equations (1.4).

Eisenhart* has shown that the condition $g \neq 0$ and the condition that the vector normal to $V_{n}$ be not null are equivalent; hence if we denote the components of this vector by $\xi^{i}$ they may be chosen so that

$$
a_{i j} \xi^{i} \xi^{j}=e,
$$

where $e$ is plus or minus one.

The investigations we wish to make will be facilitated if instead of the general coördinate system $x^{0}, x^{1}, \cdots, x^{n}$ we make use of a coördinate system $u^{0}, u^{1}, \cdots, u^{n}$, where the $u^{0}$ coördinate of a point measures the distance of that point from $V_{n}$ along a geodesic normal to $V_{n}$ and the coordinates $u^{1}, u^{2}, \cdots, u^{n}$ are any coördinates in $V_{n}$. In these coördinates we find that $\dagger$

$$
c_{11}=e, \quad c_{0 \alpha}=c_{\alpha 0}=0
$$$$
(\alpha=1,2, \cdots, n),
$$

where $e$ is defined by equation (4.7), and hence the form (1.1) becomes

$$
\varphi=e\left(d u^{0}\right)^{2}+c_{\alpha \beta} d u^{\alpha} d u^{\beta} .
$$

* Eisenhart, loc. cit., p. 144.

† Cf. Bianchi, 2d edition, loc. cit., p. 336, when the form (1.1) is assumed positive definite. Eisenhart, loc. cit., p. 146, treats the general case. 
The equation of $V_{n}$ in these coördinates is $u^{0}=0$, and equations (4.4) become

$$
g_{\alpha \beta}=c_{\alpha \beta}\left(0, u^{1}, \cdots, u^{n}\right) \quad(\alpha, \beta=1,2, \cdots, n) .
$$

If $c^{i j}$ is the cofactor of $c_{i j}$ in the determinant $c=\left|c_{i j}\right|$ divided by $c$ itself,

$$
g^{\alpha \beta}=c^{\alpha \beta}\left(0, u^{1}, \cdots, u^{n}\right) \quad(\alpha, \beta=1,2, \cdots, n) .
$$

We observe further that the components in the coördinates $u^{i}$ of the vector normal to $V_{n}$ are given by

$$
\xi^{0}=1, \quad \xi^{\alpha}=0 \quad(\alpha=1,2, \cdots, n) .
$$

5. Bianchi considers the variation in the fundamental form of $V_{n+3}$ as one passes from $V_{n}$ to a hypersurface geodesically parallel to $V_{n}$. Letting $\epsilon$ be the constant distance between these two hypersurfaces, he finds that ${ }^{*}$

$$
\delta \varphi=\epsilon\left(\frac{\partial c_{\alpha \beta}}{\partial u^{0}}\right)_{0} d u^{\alpha} d u^{\beta},
$$

and he defines functions $\Omega_{\alpha \beta}$ by the equations

$$
\Omega_{\alpha \beta}=-\left.\frac{1}{2} \frac{\partial c_{\alpha \beta}}{\partial u^{0}}\right|_{u 0=0} .
$$

The two quadratic forms that we are thus led to, $g_{\alpha \beta} d u^{\alpha} d u^{\beta}$ and $\Omega_{\alpha \beta} d u^{\alpha} d u^{\beta}$, known respectively as the first and second fundamental forms of the hypersurface, are of prime importance in the study of the variety. But their coefficients $g_{\alpha \beta}$ and $\Omega_{\alpha \beta}$ are only the first two terms in the expansion of $c_{\alpha \beta}$ in a power series in $u^{0}$,

$$
c_{\alpha \beta}=g_{\alpha \beta}-2 \Omega_{\alpha \beta} u^{0}+\stackrel{(2)}{c_{\alpha \beta}\left(u^{0}\right)^{2}+}+\stackrel{(3)}{c_{\alpha \beta}}\left(u^{0}\right)^{3}+\cdots,
$$

so it is natural to inquire into the nature of the remaining terms.

That the functions $c_{\alpha \beta}^{(m)}$ are of an intrinsic nature is shown by the following theorem:

The functions $c_{\alpha \beta}^{(m)}$ are tensors in the hypersurface and invariants in the space.

The proof of the first statement is a direct consequence of the theorem of $\S 3$. For the second part we observe that if we had, in $V_{n+1}$, two general coördinate systems $x$ and $\bar{x}$ it would follow that

$$
a_{i j} \frac{\partial x^{i}}{\partial u^{\alpha}} \frac{\partial x^{j}}{\partial u^{\beta}}=\left(\bar{a}_{r s} \frac{\partial \bar{x}^{r}}{\partial x^{i}} \frac{\partial \bar{x}^{s}}{\partial x^{i}}\right) \frac{\partial x^{i}}{\partial u^{\alpha}} \frac{\partial x^{j}}{\partial u^{\beta}}=\bar{a}_{r s} \frac{\partial \bar{x}^{r}}{\partial u^{\alpha}} \frac{\partial \bar{x}^{s}}{\partial u^{\beta}},
$$

\footnotetext{
* Bianchi, 2d edition, loc. cit., p. 360.
} 
and hence

$$
c_{\alpha \beta}=a_{i j} \frac{\partial x^{i}}{\partial u^{\alpha}} \frac{\partial x^{i}}{\partial u^{\beta}} \quad(\alpha, \beta=1,2, \cdots, n)
$$

is an invariant in $V_{n+1}$, and consequently the functions $c_{\alpha \beta}^{(m)}$ are invariants in $V_{n+1}$.

6. We devote the remainder of this paper to the determination of these tensors and to some applications to particular spaces and hypersurfaces.

From the definitions of the Christoffel symbols in equations (1.5) and (1.6), we have that, for the fundamental form (4.9),

(6.1) $[00,0]=[00, \alpha]=[0 \alpha, 0]=0, \quad[0 \alpha, \beta]=\frac{1}{2} \frac{\partial c_{\alpha \beta}}{\partial u^{0}}(\alpha, \beta=1,2, \cdots, n)$ and hence that

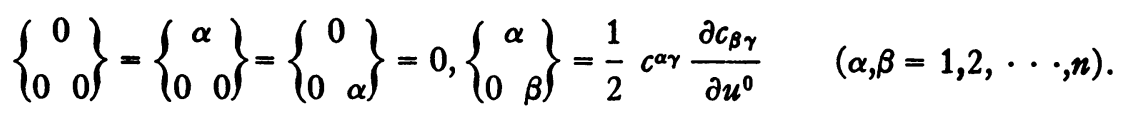

If we evaluate $R_{0 \alpha \beta 0}$ from equations (1.7), we are able to solve for the second derivative, obtaining that

$$
\frac{\partial^{2} c_{\alpha \beta}}{\left(\partial u^{0}\right)^{2}}=2 R_{0 \alpha \beta 0}+\frac{1}{2} c^{\gamma \delta} \frac{\partial c_{\alpha \gamma}}{\partial u^{0}} \frac{\partial c_{\beta \delta}}{\partial u^{0}} \quad(\alpha, \beta=1,2, \cdots, n),
$$

and hence, by virtue of (4.11) and (5.2),

$$
c_{\alpha \beta}^{(2)}=R_{0 \alpha \beta 0}+\Omega_{\alpha}^{\gamma} \Omega_{\beta \gamma},
$$

where

$$
\Omega_{\alpha}^{\gamma}=g^{\gamma \beta} \Omega_{\alpha \beta}=g^{\gamma \beta} \Omega_{\beta \alpha},
$$

and it is understood in (6.4) that $R_{0 \alpha \beta 0}$ is evaluated on the hypersurface. For a general coördinate system, $x^{0}, x^{1}, \cdots, x^{n}$, in the space, the third fundamental tensor is given by

$$
\stackrel{(2)}{c_{\alpha \beta}}=\bar{R}_{i j k l} \frac{\partial x^{j}}{\partial u^{\alpha}} \frac{\partial x^{k}}{\partial u^{\beta}} \xi^{i} \xi^{l}+\Omega_{\alpha}^{\gamma} \Omega_{\beta \gamma},
$$

where the $\bar{R}$ 's here are the Riemann tensors with respect to the fundamental form of the $x$ 's.

We can obtain $\partial^{3} c_{\alpha \beta} /\left(\partial u^{0}\right)^{3}$ by differentiating equations (6.3),

$$
\frac{\partial^{3} c_{\alpha \beta}}{\left(\partial u^{0}\right)^{3}}=2 \frac{\partial}{\partial u^{0}} R_{0 \alpha \beta 0}+\frac{1}{2} \frac{\partial}{\partial u^{0}}\left(c^{\gamma \delta} \frac{\partial c_{\alpha \gamma}}{\partial u^{0}} \frac{\partial c_{\beta \delta}}{\partial u^{0}}\right) \text {. }
$$


The ordinary derivative of $R_{0 \alpha \beta 0}$ has not an invariantive significance, and so it is preferable to replace it by the covariant derivative $R_{0 \alpha \beta 0,0}$. From the definition, simplified by virtue of (6.2), we obtain that

$$
\frac{\partial}{\partial u^{0}} R_{0 \alpha \beta 0}=R_{0 \alpha \beta 0,0}+\frac{1}{2} c^{\mu \nu} \frac{\partial c_{\alpha \nu}}{\partial u^{0}} R_{0 \mu \beta 0}+\frac{1}{2} c \mu \nu \frac{\partial c_{\beta \nu}}{\partial u^{0}} R_{0 \alpha \nu 0} .
$$

Furthermore, from

$$
c^{\alpha i} c_{i \beta}=c^{\alpha \gamma} c_{\gamma \beta}=\delta_{\beta}^{\alpha} \quad(\alpha, \beta=1,2, \cdots, n)
$$

it follows that

$$
c^{\alpha \gamma} \frac{\partial c_{\gamma \beta}}{\partial u^{0}}=-c_{\gamma \beta} \frac{\partial c^{\alpha \gamma}}{\partial u^{0}} .
$$

Hence equations (6.3) are equivalent to

$$
c^{\delta \gamma} \frac{\partial^{2} c_{\alpha \gamma}}{\left(\partial u^{0}\right)^{2}}=2 R_{0 \alpha 0}^{\delta}-\frac{1}{2} \frac{\partial c_{\alpha \gamma}}{\partial u^{0}} \frac{\partial c^{\delta \gamma}}{\partial u^{0}},
$$

from which we obtain that

$$
\frac{\partial}{\partial u^{0}}\left(c^{\gamma \delta} \frac{\partial c_{\alpha \gamma}}{\partial u^{0}}\right)=2 R_{0 \alpha 0}^{\delta}+\frac{1}{2} \frac{\partial c_{\alpha \gamma}}{\partial u^{0}} \frac{\partial c^{\delta \gamma}}{\partial u^{0}} .
$$

Substituting (6.8), (6.11), and (6.3) in (6.7) and making a slight simplification due to the equalities

$$
R_{0 \alpha \beta 0}=R_{0 \beta \alpha 0}, \quad R_{0 \alpha 0}^{\beta}=c^{\beta \gamma} R_{0 \gamma \alpha 0}=c^{\beta \gamma} R_{0 \alpha \gamma 0},
$$

we obtain that

$$
\frac{\partial^{3} c_{\alpha \beta}}{\left(\partial u^{0}\right)^{3}}=2 R_{0 \alpha \beta 0,0}+2 c^{\mu \nu}\left(\frac{\partial c_{\mu \alpha}}{\partial u^{0}} R_{0 \nu \beta 0}+\frac{\partial c_{\mu \beta}}{\partial u^{0}} R_{0 \alpha \nu 0}\right) .
$$

To obtain the fourth derivative in intrinsic form, we observe that because of equation (6.2) we are able to write

$$
\frac{\partial R_{0 \alpha \beta 0,0}}{\partial u^{0}}=R_{0 \alpha \beta 0,00}+\frac{1}{2} c^{\mu \nu}\left\{\frac{\partial c_{\nu \alpha}}{\partial u^{0}} R_{0 \mu \beta 0,0}+\frac{\partial c_{\nu \beta}}{\partial u^{0}} R_{0 \alpha \mu 0,0}\right\} .
$$

Furthermore, in the consideration of the term

$$
c^{\mu \nu} \frac{\partial c_{\mu \alpha}}{\partial u^{0}} R_{0, \beta 0}
$$


of equations (6.13), differentiation of the first two members by application of equation (6.11) introduces, among others, the term

$$
\frac{\partial c_{\mu \alpha}}{\partial u^{0}} \frac{\partial c^{\mu \nu}}{\partial u^{0}} R_{0, \beta 0},
$$

while the application of formula (6.8) to the differentiation of $R_{0 \times \beta 0}$ introduces

$$
c^{\mu \nu} \frac{\partial c_{\mu \alpha}}{\partial u^{0}} c^{\sigma \tau} \frac{\partial c_{\tau} \nu}{\partial u^{0}} R_{0 \sigma \beta 0} ;
$$

because of equations (6.10) these expressions are equal numerically, but opposite in sign; the two similar terms obtained in the differentiation of

$$
c^{\mu \nu} \frac{\partial c_{\nu \beta}}{\partial u^{0}} R_{0 \alpha \nu 0}
$$

likewise cancel each other. These are the only cancellations occurring, so that we have

$$
\begin{aligned}
\frac{\partial^{4} c_{\alpha \beta}}{\left(\partial u^{0}\right)^{3}}= & 2 R_{0 \alpha \beta 0,00}+8 R_{0 \alpha 0}^{\mu} R_{0 \mu \beta 0} \\
& +3 c^{\nu \mu}\left(\frac{\partial c_{\mu \alpha}}{\partial u^{0}} R_{0 \nu \beta 0,0}+\frac{\partial c_{\mu \beta}}{\partial u^{0}} R_{0 \alpha \nu 0,0}\right) \\
& +2 R_{0 \lambda \mu 0} c^{\lambda \sigma} \frac{\partial c_{\sigma \alpha}}{\partial u^{0}} c^{\mu \tau} \frac{\partial c_{\tau \beta}}{\partial u^{0}} .
\end{aligned}
$$

Similarly, we obtain $\partial^{5} c_{\alpha \beta} /\left(\partial u^{0}\right)^{5}$ by differentiation of (6.15), but instead, let us turn to the general derivative to evaluate it.

7. For the consideration of the general coefficient it will be desirable to introduce a slightly more convenient notation, in part necessary for the sake of clearness. We can see that the covariant derivatives of $R_{0 \alpha \beta 0}$ with respect to $u^{0}$ and of all orders will appear in the following pages and we shall denote by $R_{0 \alpha \beta 0,[l]}$ and $R_{0 \beta 0,[l]}^{\alpha}$ the $l$ th covariant derivative with respect to $u^{0}$ of $R_{0 \alpha \beta 0}$ and $R_{0 \beta 0}^{\alpha}$ respectively. Thus,

$$
R_{0 \alpha \beta 0,[3]} \equiv R_{0 \alpha \beta 0,000}, \quad R_{0 \beta 0,[4]}^{\alpha} \equiv R_{0 \beta 0,0000}^{\alpha} .
$$

For symmetry in our formulas, we shall interpret $R_{0 \alpha \beta 0 \text {, [0] }}$ as merely $R_{0 \alpha \beta 0}$, that is,

$$
R_{0 \alpha \beta 0,[0]} \equiv R_{0 \alpha \beta 0}, \quad R_{0 \beta 0,[0]}^{\alpha} \equiv R_{0 \beta 0}^{\alpha}
$$


With this notation we are able to write the general formula suggested by equations (6.8) and (6.14), namely

$$
\text { (7.1) } \frac{\partial}{\partial u^{0}} R_{0 \alpha \beta 0,[l]}=R_{0 \alpha \beta 0,[l+1]}+\frac{1}{2} c^{\mu \nu}\left(\frac{\partial c_{\nu \alpha}}{\partial u^{0}} R_{0 \mu \beta 0,[l]}+\frac{\partial c_{\nu \beta}}{\partial u^{0}} R_{0 \alpha \mu 0,[l]}\right) \text {. }
$$

This follows immediately from the definition of covariant differentiation when the Christoffel symbols are subject to equations (6.2). Similarly we have for the covariant derivative of the four-index symbol of Riemann of the second kind, that

$$
\text { (7.2) } \frac{\partial R_{0 \beta 0,[l]}^{\alpha}}{\partial u^{0}}=R_{0 \beta 0,[l+1]}^{\alpha}+\frac{1}{2} c^{\mu \nu} \frac{\partial c_{\nu \beta}}{\partial u^{0}} R_{0 \mu 0,[l]}^{\alpha}-\frac{1}{2} c^{\mu \alpha} \frac{\partial c_{\mu \nu}}{\partial u^{0}} R_{0 \beta 0,[l]}^{\nu} \text {. }
$$

In the following discussion there will arise contracted products or several $R$ 's. We introduce the notation $R_{\left[k_{1} k_{2} \ldots k_{N}\right] \alpha \beta}$, defining it by the equation

$$
\text { (7.3) } R_{\left[k_{1} \cdots k_{N}\right] \alpha \beta} \equiv R_{0 \mu_{2},\left[k_{1}\right]}^{\mu_{1}} R_{0 \mu_{3} 0,\left[k_{2}\right]}^{\mu_{2}} \cdots R_{0 \mu_{N-1}, l_{N-2}}^{\left.\mu_{N-2}\right]} R_{0 \alpha 0,\left[k_{N-1}\right]}^{\mu_{N-1}} R_{0 \mu_{1} \beta 0,\left[k_{N}\right]} \text {. }
$$

We note that $N$ may take on any value $2,3, \cdots$, while the $k$ 's may take on $0,1,2, \cdots$. For further clarity let us observe that

$$
\begin{aligned}
R_{[01] \alpha \beta} & =R_{0 \alpha 0}^{\mu} R_{0 \mu \beta 0,0}, \\
R_{[210] \alpha \beta} & =R_{0 \nu 0,00}^{\mu} R_{0 \alpha 0,0}^{\prime} R_{0 \mu \beta 0} .
\end{aligned}
$$

From the definition, from equations (6.12) and from the equations derived from (6.12) by covariant differentiation, it follows that

$$
R_{\left[k_{1} k_{2} \ldots k_{N}\right] \alpha \beta} \equiv R_{\left[k_{N-2} k_{N-3} \cdots k_{1} k_{N} k_{N-1}\right] \beta \alpha} .
$$

If we differentiate equations (7.3) covariantly with respect to $u^{0}$, we find that

(7.5) Covariant Deriv. of $R_{\left[k_{1} \cdots k_{N}\right] \alpha \beta}=\sum_{i=1}^{N} R_{\left[k_{1} \cdots k_{i-1} k_{i}+1 k_{i+1} \cdots k_{N}\right] \alpha \beta}$.

Again, if we evaluate $\left(\partial / \partial u^{0}\right) R_{\left[k_{1}\right.} \cdots k_{N] \alpha \beta}$ by means of equations (7.1) and (7.2) we find that the term $R_{0 \mu_{i+1} \mu^{0},\left[k_{i}\right]}^{\mu_{1}}$ of $R$ contributes the single term

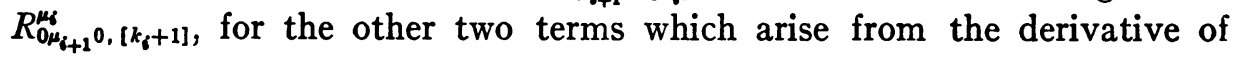
$R_{0_{i+1}, j_{i},\left[k_{i}\right]}^{\mu_{i}}$ are cancelled by two similar terms one of which arises from the differentiation of $R_{\left.0 \mu_{i} j_{0}, k_{k-1}\right]}^{\mu_{1}-1}$ and the other from the differentiation of $R_{0_{i+2}, \mu_{i+1}+1}^{\left.\mu_{i+1}\right]}$; hence we have that

$$
\begin{aligned}
& \frac{\partial}{\partial u^{0}} R_{\left[k_{1} \ldots k_{N}\right] \alpha \beta}=\sum_{i=1}^{n} R_{\left[k_{1} \cdots k_{i-1} k_{i}+1 k_{i+1} \cdots k_{N}\right] \alpha \beta} \\
& +\frac{1}{2} c \mu \nu \frac{\partial c_{\nu \alpha}}{\partial u^{0}} R_{\left[k_{1} \cdots k_{N}\right] \alpha \mu}+\frac{1}{2} c^{\mu \nu} \frac{\partial c_{\nu \beta}}{\partial u^{0}} R_{\left[k_{1} \cdots k_{N}\right] \alpha \mu \cdot}
\end{aligned}
$$




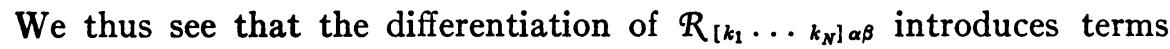
of the type

$$
c^{\mu \nu} \frac{\partial c_{\nu \beta}}{\partial u^{0}} R_{\left[k_{1} \ldots k_{N}\right] \alpha \mu}
$$

and accordingly we should like to have the expression for the derivative of a term of this type. Equations (6.11) and (7.6) enable us to obtain it; here again there will be a cancellation, the second term of (6.11) annulling one of the terms introduced by the differentiation of $R$. Observing that $R_{000}^{\mu} R_{\left[k^{1} \cdots k_{N}\right] \alpha \mu}=R_{\left[k_{N} k_{1} \cdots k_{N-1} 0\right] \alpha \beta}$, we are able to write

$$
\begin{gathered}
\frac{\partial}{\partial u^{0}}\left\{c^{\mu \nu} \frac{\partial c_{\nu \beta}}{\partial u^{0}} R_{\left[k_{1} \ldots k_{N}\right] \alpha \beta}\right\}=2 R_{\left[k_{N} k_{1} \ldots k_{N-1} 0\right] \alpha \beta} \\
+c^{\mu \nu} \frac{\partial c_{\nu \beta}}{\partial u^{0}} \sum_{i=1}^{N} R_{\left[k_{1} \ldots k_{i-1} k_{i}+1 k_{i+1} \ldots k_{N}\right] \alpha \beta} \\
+\frac{1}{2} c^{\mu \nu} c^{\sigma \sigma r} \frac{\partial c_{\nu \beta}}{\partial u^{0}} \frac{\partial c_{\sigma \alpha}}{\partial u^{0}} R_{\left[k_{1} \ldots k_{N}\right]+\mu} .
\end{gathered}
$$

Either from equations (7.4) or directly, we have the similar result that

$$
\begin{gathered}
\frac{\partial}{\partial u^{0}}\left\{c^{\mu \nu} \frac{\partial c_{\nu \beta}}{\partial u^{0}} R_{\left[k_{1} \ldots k_{N}\right] \mu \alpha}\right\}=2 R_{\left[k_{1} \ldots k_{N-1} 0 k_{N}\right] \beta \alpha} \\
+c^{\nu \mu} \frac{\partial c_{\nu \beta}}{\partial u^{0}} \sum_{i=1}^{N} R_{\left[k_{1} \ldots k_{i-1} k_{i}+1 k_{i+1} \ldots k_{N}\right] \mu \alpha} \\
+\frac{1}{2} c^{\mu \nu} c^{\lambda \sigma} \frac{\partial c_{\nu \beta}}{\partial u^{0}} \frac{\partial c_{\sigma \alpha}}{\partial u^{0}} R_{\left[k_{1} \ldots k_{N}\right] \mu \lambda .} .
\end{gathered}
$$

Finally, we observe that we need a formula for the derivative of

$$
c^{\lambda \sigma} c^{\mu \nu} \frac{\partial c_{\sigma \alpha}}{\partial u^{0}} \frac{\partial c_{\nu \beta}}{\partial u^{0}} R_{\left[k_{1} \ldots k_{s}\right] \lambda_{\mu}} .
$$

This we obtain from a combination of equations (6.11) with either (7.7) or (7.8). Here again the term arising from the second term of (6.11) will cancel the one arising from the last term of equations (7.7) (or (7.8)). The result therefore is

$$
\begin{aligned}
& \frac{\partial}{\partial u^{0}}\left\{c^{\lambda \sigma^{\mu} c_{\nu}} \frac{\partial c_{\sigma \alpha}}{\partial u^{0}} \frac{\partial c_{\nu \beta}}{\partial u^{0}} R_{\left(k_{1} \ldots k_{N}\right] \lambda_{\mu}}\right\} \\
& =2 c^{\mu \nu} \frac{\partial c_{\nu \beta}}{\partial u^{0}} R_{\left[k_{1} \ldots k_{N-1} 0 k_{N}\right] \alpha \mu}+2 c^{\lambda \sigma} \frac{\partial c_{\sigma \alpha}}{\partial u^{0}} R_{\left[k_{N} k_{1} \ldots k_{N-1} 0\right] \lambda \beta} \\
& +c^{\lambda \epsilon} c^{\mu \nu} \frac{\partial c_{\sigma \alpha}}{\partial u^{0}} \frac{\partial c_{\nu \beta}}{\partial u^{0}} \sum_{i=1}^{N} R_{\left[k_{1} \ldots k_{i-1} k_{i+1} k_{i+1} \ldots k_{\alpha U}\right] \lambda_{\mu} .}
\end{aligned}
$$


The formulas here obtained, namely (7.6), (7.7), (7.8) and (7.9), indicate the general character of $\partial^{m} c_{\alpha \beta} /\left(\partial u^{0}\right)^{m}$ and lead us to write the following expression :

$$
\begin{aligned}
\frac{\partial^{m} c_{\alpha \beta}}{\left(\partial u^{0}\right)^{m}} & =2 R_{0 \alpha \beta 0,[m-2]}+\sum_{\sigma, N}^{m} D_{\sigma_{1} \sigma_{2} \ldots \sigma_{N}}^{(m)} R_{\left[\sigma_{1} \ldots \sigma_{N}\right] \alpha \beta} \\
& +c^{\lambda \mu} \frac{\partial c_{\lambda \alpha}}{\partial u^{0}}\left\{(m-1) R_{0 \mu \beta 0,[m-3]}+\sum_{\tau, N}^{m-1} E_{\tau_{1} \ldots \tau_{N}}^{(m)} R_{\left[\tau_{1} \ldots \tau_{N}\right] \mu \beta}\right\} \\
& +c^{\lambda \mu} \frac{\partial c_{\lambda \alpha}}{\partial u^{0}}\left\{(m-1) R_{0 \mu \alpha 0,[m-3]}+\sum_{\tau, N}^{m-1} \bar{E}_{\tau_{1} \ldots \tau_{N}}^{(m)} R_{\left[\tau_{1} \ldots \tau_{N}\right] \mu \alpha}\right\} \\
+ & c^{\nu \mu} c^{\nu \sigma} \frac{\partial c_{\lambda \alpha}}{\partial u^{0}} \frac{\partial c_{\sigma \beta}}{\partial u^{0}}\left\{\frac{m(m-3)}{2} R_{0 \mu \nu,[m-4]}+\sum_{\tau_{, N N}}^{m-2} F_{\pi_{1} \ldots \pi_{N}}^{(m)} R_{\left[\pi_{1} \ldots \pi_{N}\right] \mu \nu}\right\}
\end{aligned}
$$

where the $D$ 's, $E$ 's, and $F^{\prime}$ 's are constants to be determined, and where $\sum_{\sigma, N}^{m}$ represents the sum over $N=2,3,4 \cdots$ and $\sigma_{i}=0,1,2, \cdots$ and such that $2 N+\sigma_{1}+\sigma_{2}+\cdots+\sigma_{N}=m$.

Since $\partial^{m} C_{\alpha \beta} /\left(\partial u^{0}\right)^{m}$ is symmetric in $\alpha$ and $\beta$, the right hand side of (7.10) must be also, and independently of the $R$ 's; this gives us conditions which by virtue of equation (7.4) become

$$
\begin{aligned}
& D_{\sigma_{1} \ldots \sigma_{N}}^{(m)}=D_{\sigma_{N-2} \sigma_{N-3} \ldots \sigma_{1} \sigma_{N} \sigma_{N-1}}^{(m)}, \\
& \bar{E}_{\tau_{1} \ldots \tau_{N}}^{(m)}=E_{\tau_{1} \ldots \tau_{N}}^{(m)}, \\
& F_{\pi_{1} \ldots \pi_{N}}^{(m)}=F_{\pi_{N-2} \cdots \pi_{1} \pi_{2} \pi_{N-1}}^{(m)} .
\end{aligned}
$$

If we form $\partial^{m+1} c_{\alpha \beta} /\left(\partial u^{0}\right)^{m+1}$ by differentiating (7.10) with respect to $u^{0}$ making use of the four fundamental formulas (7.6), (7.7), (7.8) and (7.9) and of equations (7.4) and (7.11), and compare the result with the equation obtained from (7.10) by writing $m+1$ for $m$, we obtain the justification for the precise numerical coefficients of (7.10) and at the same time we find recursion formulas for the $D$ 's, $E$ 's, and $F$ 's. To facilitate this differentiation and the collection of the results, we observe that there are three types of terms in (7.10), those in which $\partial c_{\mu \nu} / \partial u^{0}$ does not appear, those linear in these derivatives, and finally, those quadratic in them. Those of the first type can arise only from those of the first or second type, those of the second type arise from all three types, but those of the third type arise only from those of the second and third types. With these observations the recursion formulas follow immediately from equations (7.6) to (7.9); they are 


$$
D_{\rho_{1} \ldots \rho_{N}}^{m+1}=\sum_{i=1}^{N} D_{\rho_{1} \ldots \rho_{i-1} \rho_{i-1} \rho_{i+1} \ldots \rho_{N}}^{(m)}+2(m-1) \delta_{\rho_{N}}^{m-3} \delta_{\rho_{N-1}}^{0}
$$

$$
\begin{aligned}
& +2 E_{\rho_{1} \ldots \rho_{N-2} \rho_{N} \delta_{\rho_{N-1}}^{(m)}}^{0}+2(m-1) \delta_{\rho_{N-1}}^{m-3} \delta_{\rho_{N}}^{0} \\
& +2 E_{\rho_{N-2} \rho_{N-3} \ldots \rho_{1} \rho_{N} \delta_{\rho_{N}}^{0}}^{(m)} \\
& E_{\sigma_{1} \cdots \sigma_{N}}^{(m+1)}=\frac{1}{2} D_{\sigma_{1} \ldots \sigma_{N}}^{(m)}+\sum_{i=1}^{N} E_{\sigma_{1} \ldots \sigma_{i}-1 \ldots \sigma_{N}}^{(m)} \\
& +m(m-3) \delta_{\sigma_{N-1} \delta_{\sigma_{N}}}^{m-4}+2 F_{\sigma_{1} \ldots \sigma_{N-1} \sigma_{1} \delta_{\sigma_{N}}^{0}}, \\
& F_{\tau_{1} \ldots \tau_{N}}^{(m+1)}=\sum_{i=1}^{N} F_{\tau_{1} \ldots \tau_{i-1} \ldots \tau_{N}}^{(m)}+\frac{1}{2} E_{\tau_{1} \ldots \tau_{N}}^{(m)} \\
& \quad+\frac{1}{2} E_{\tau_{N-2} \tau_{N-3} \ldots \tau_{1} \tau_{N} \tau_{N-1}}^{(m)}
\end{aligned}
$$

where the $\delta$ 's are defined by equations (1.4) and where a $D, E$, or $F$ with a negative subscript is to be interpreted as zero.

Summing up these results in the form of a theorem, we have the following:

The mth derivative of $c_{\alpha \beta}$ with respect to $u^{0}$ is expressible as a non-homogeneous polynomial of the second degree in the first derivatives $\partial c_{\lambda_{\mu}} / \partial u^{0}$ $(\lambda, \mu=1,2, \cdots, n)$ whose coefficients are polynomials in the Riemann tensor and its covariant derivatives of orders less than and equal to $m-2$.

8. The fundamental tensors in questions, $c_{\alpha \beta}^{(m)}$, are the derivatives evaluated on the hypersurface $u^{\theta}=0$. We recall the definition of $\Omega_{\alpha \beta}$ and $\Omega_{\beta}^{\alpha}$ from equations (5.2) and (6.5) respectively, and the relations (4.10) and (4.11) between $g_{\alpha \beta}$ and $c_{\alpha \beta}$. We observe further that if $\bar{R}_{i j k l,}, \ldots$ is the $R$ in a general coördinate system, we must have

$$
R_{0 \alpha \beta 0,[l]}=\bar{R}_{h i j k, r_{1} \ldots r_{l}} \frac{\partial x^{i}}{\partial u^{\alpha}} \frac{\partial x^{j}}{\partial u^{\beta}} \xi^{k} \xi^{k} \xi^{r_{1}} \cdots \xi^{r l}
$$

where $\xi^{i}=\partial x^{i} / \partial u^{0}$ is the normal vector to $V_{n}$. Hence this theorem follows :

The fundamental tensors of a hypersurface are expressible in terms of the first two fundamental tensors of the hypersurface and of the Riemann tensor of the space and its covariant derivatives, evaluated on the hypersurface.

For reference we shall give the explicit values of the first five of these tensors, the first three being obtained directly from equations (6.4), (6.13) and (6.15) and the others by repeated application of the recursion formulas 
(7.12), (7.13) and $(7.14)^{*}$ :

$$
\begin{aligned}
c_{\alpha \beta}^{(2)}= & R_{0 \alpha \beta 0}+\Omega_{\alpha}^{\gamma} \Omega_{\gamma \beta}, \\
c_{\alpha \beta}^{(2)}= & \frac{2}{3 !} R_{0 \alpha \beta 0,0}-\frac{4}{3 !}\left(R_{0 \alpha \gamma 0} \Omega_{\beta}^{\gamma}+R_{0 \gamma \beta 0} \Omega_{\alpha}^{\gamma}\right), \\
c_{\alpha \beta}^{(())}= & \frac{1}{4 !}\left(2 R_{0 \alpha \beta 0,00}+8 R_{0 \alpha 0}^{\mu} R_{0 \mu \beta 0}-6 R_{0 \alpha \mu 0,0} \Omega_{\beta}^{\mu}\right. \\
& \left.\quad-6 R_{0 \beta \mu 0,0} \Omega_{\alpha}^{\mu}+8 R_{0 \mu \lambda 0} \Omega_{\alpha}^{\mu} \Omega_{\beta}^{\lambda}\right), \\
c_{\alpha \beta}^{(b)}= & \frac{1}{5 !}\left\{2 R_{0 \alpha \beta 0,000}+14 R_{0 \alpha 0}^{\mu} R_{0 \mu \beta 0,0}+14 R_{0 \alpha 0,0}^{\mu} R_{0 \mu \beta 0}\right. \\
& \quad-8 \Omega_{\alpha}^{\mu}\left(R_{0 \mu \beta 0,00}+2 R_{0 \mu 0}^{\prime} R_{0 \gamma \beta 0}\right)-8 \Omega_{\beta}^{\mu}\left(R_{0 \mu \alpha 0,00}\right. \\
& \left.\left.\quad+2 R_{0 \mu 0}^{\prime} R_{0, \beta 0}\right)+20 \Omega_{\alpha}^{\lambda} \Omega_{\beta}^{\mu} R_{0 \lambda \mu 0,0}\right\} .
\end{aligned}
$$

For $m=6$, we find

$$
\begin{aligned}
\stackrel{(6)}{c_{\alpha \beta}=\frac{1}{6 !}} & \left(2 R_{0 \alpha \beta 0,0000}+22 R_{0 \alpha 0}^{\mu} R_{0 \mu \beta 0,00}+28 R_{0 \alpha 0,0}^{\mu} R_{0 \mu \beta 0,0}\right. \\
& \left.+22 R_{0 \alpha 0,00} R_{0 \mu \beta 0}+32 R_{0 \nu 0}^{\mu} R_{0 \alpha 0}^{\prime} R_{0 \mu \beta 0}\right) \\
& -\frac{10}{6 !} \Omega_{\alpha}^{\mu}\left(R_{0 \mu \beta 0,000}+3 R_{0 \mu 0}^{\prime} R_{0, \beta 0,0}+5 R_{0 \mu 0,0}^{\prime} R_{0, \beta 0}\right) \\
& -\frac{10}{6 !} \Omega_{\beta}^{\mu}\left(R_{0 \mu \alpha 0,000}+3 R_{0 \mu 0}^{\prime} R_{0 \nu \alpha 0,0}+5 R_{0 \mu 0,0}^{\prime} R_{0, \alpha 0}\right) \\
& +\frac{1}{6 !} \Omega_{\alpha}^{\lambda} \Omega_{\beta}^{\mu}\left(36 R_{0 \lambda \mu 0,00}+32 R_{0 \lambda 0}^{\prime} R_{0 \nu \mu 0}\right) .
\end{aligned}
$$

\section{PART III}

9. We finally turn to the consideration of the tensors, and to obtaining some general properties. We have already observed in $\S 5$ the geometrical interpretation Bianchi obtained for the functions $\Omega_{\alpha \beta}$. We shall here give another, one which has its analogue in 3-dimensional differential geometry.

Consider the hypersurface generated by the geodesics of $V_{n+1}$ tangent to $V_{n}$ at an arbitrary, but fixed point $P$ of $V_{n}$; such a hypersurface we call the tangent geodesic hypersurface to $V_{n}$ at $P$. We prove the following theorem :

- These values of $c_{\alpha \beta}^{(m)}$ were found by me first by making use of general coördinates. However, at the suggestion of G. Y. Rainich I made use of the particular coördinate system introduced in $\$ 4$, and that not only enabled me to obtain my previous results more readily, but also made possible the determination of the general expression for $c_{a \beta}^{(m)}$ (equations (7.10)). 
If $\bar{V}_{n}$ is the tangent geodesic hypersurface to $V_{n}$ at a point $P$ and if $P^{\prime}$ is a point of $\bar{V}_{n}$ neighboring to $P$, then the distance from $P^{\prime}$ to $V_{n}$ is given, except for terms of higher order, by $-\frac{1}{2} e \Omega_{\alpha \beta} d u^{\alpha} d u^{\beta}$.

The proof is very direct. Let us find a parametric representation of the tangent geodesic hypersurface. The geodesic through the point with coordinates $\left(u_{(0)}^{0}, u_{(0)}^{1}, \cdots, u_{(0)}^{n}\right)$ and direction $\left(\zeta^{0}, \zeta^{1}, \cdots, \zeta^{n}\right)$ at this point has for its equation, when the coördinates of the space are the $u$ 's, the infinite series

(9.1) $u^{i}=u_{(0)}^{i}+\zeta^{i} s-\frac{1}{2}\left\{\begin{array}{c}i \\ r s\end{array}\right\} \zeta^{r} \zeta^{s} s^{2}-\frac{1}{3 !} \bar{\Gamma}_{\text {ret }}^{i} \zeta^{r} \zeta^{s} \zeta^{3} s^{3}-\cdots(i=0,1, \cdots n)$,

where the functions $\bar{\Gamma}$ are formed with respect to the linear element (4.9) in precisely the way in which the $\Gamma$ 's of equations (2.10) are formed with respect to the linear element $(1.1)$ and are evaluated for the point $u_{(0)}$. If we take for $u_{(0)}$ the point $P$ with coördinates $\left(0, u_{(0)}^{\mathbf{1}}, \boldsymbol{u}_{(0)}^{\mathbf{2}}, \cdots, \boldsymbol{u}_{(0)}^{\mathbf{n}}\right)$ ? and for the direction one with components $\left(0, \zeta^{1}, \zeta^{2}, \cdots, \zeta^{n}\right)$, the geodesic is tangent to $V_{n}$ at $P$. When we set $v^{\alpha}=\zeta^{\alpha} s$, equations (9.1) become

$$
\begin{aligned}
& u^{0}=-\frac{1}{2}\left\{\begin{array}{c}
0 \\
\beta \gamma
\end{array}\right\} v^{\beta} v^{\gamma}-\frac{1}{3 !} \bar{\Gamma}_{\beta \gamma \delta v^{\beta} v^{\gamma} v^{\delta}}^{0}-\cdots \\
& u^{\alpha}=u_{(0)}^{\alpha}+v^{\alpha}-\frac{1}{2}\left\{\begin{array}{c}
\alpha \\
\beta \gamma
\end{array}\right\} v^{\beta} v^{\gamma}-\cdots .
\end{aligned}
$$

These equations, when we regard $v^{1}, v^{2}, \cdots, v^{n}$ as parameters, define the tangent geodesic hypersurface. Since the $u^{0}$ coördinate of a point $\left(u^{0}\right.$, $\left.u^{1}, \cdots, u^{n}\right)$ is the distance from the point to the hypersurface $V_{n}$, the required distance, $D$, from a point $P^{\prime}$ of the tangent geodesic hypersurface to $V_{n}$ is given by

$$
D=-\frac{1}{2}\left\{\begin{array}{c}
0 \\
\beta \gamma
\end{array}\right\} v^{\beta} v^{\gamma} .
$$

But $v^{\beta}$ is $d u^{\beta}$ except for terms of higher order; furthermore, by virtue of equations (4.8), $\left\{\beta_{\gamma}{ }^{0}\right\}=e \Omega_{\beta_{\gamma}}$. Hence

$$
D=-\frac{1}{2} e \Omega_{\beta \gamma} d u^{\beta} d u^{\gamma} .
$$

This proves the theorem. We may make the following further observations.

The contact between a hypersurface and its tangent geodesic hypersurface is in general simple. A necessary and sufficient condition that it be of order higher than the first at a point $P$ and in the direction $\zeta$ is that

$$
\Omega_{\alpha \beta} \zeta^{\alpha} \zeta^{\beta}=0
$$


at $P$. If (9.4) holds for every direction and at every point of the hypersurface, we have

$$
\Omega_{\alpha \beta}=0 .
$$

We observe that hypersurfaces for which $\Omega_{\alpha \beta}=0$ may be classified by means of the equations

$$
c_{\alpha \beta}^{(2)}=c_{\alpha \beta}^{(3)}=\cdots=c_{\alpha \beta}^{(m)}=0, \quad c_{\alpha \beta}^{(m+1)} \neq 0 .
$$

The larger the value of $m$ for which this is true, the more nearly will the geometry of the space and the hypersurface be the same. In general a space does not contain any hypersurface for which $c_{\alpha \beta}^{(m)}=0$ for all $m$. We shall not at present carry the problems here suggested any further; instead we shall consider, in the remainder of this paper, a few special cases.

10. First suppose the form (1.1) or its equivalent (4.9) is reducible to a form with constant coefficients. Such a space we call a flat space. We observe that a flat space with a positive definite form is euclidean.

For a flat space, $R_{i j k l}$ and its covariant derivatives vanish identically; from (6.13) it follows that $c_{\alpha \beta}^{(m)}=0$ for $m \geqq 3$. For $c_{\alpha \beta}^{(m}, m<3$, equations (8.2) become

$$
c_{\alpha \beta}^{(0)}=g_{\alpha \beta}, \quad c_{\alpha \beta}^{(1)}=-2 \Omega_{\alpha \beta}, \quad c_{\alpha \beta}^{(2)}=\Omega_{\alpha \gamma} \Omega_{\beta}^{\gamma} .
$$

Bianchi* has shown that

$$
\Omega_{\alpha \delta}=-a_{i j} \frac{\partial \xi^{i}}{\partial u^{\alpha}} \frac{\partial f^{j}}{\partial u^{\delta}}-[i j, k]_{a} \frac{\partial f^{i}}{\partial u^{\alpha}} \frac{\partial f^{k}}{\partial u^{\delta}} \xi^{j},
$$

where the $f$ 's are those of equations (4.1), $[r s, t]_{a}$ are the Christoffel symbols of the space with reference to the form (1.1) and $\xi^{i}$ is the vector normal to $V_{n}$ referred to the general coördinate system of (1.1).

If the space is flat, we may take generalized cartesian coördinates, that is

$$
\begin{array}{lrl}
a_{i j}=0 & (i \neq j), \\
a_{i i}=1 & (i=0,1, \cdots, m), \\
a_{j i}=-1 & (j=m+1, \cdots, n) .
\end{array}
$$

Then we have

$$
\Omega_{\alpha \delta}=\sum_{i=0}^{m} \frac{\partial \xi^{i}}{\partial u^{\alpha}} \frac{\partial f^{i}}{\partial u^{\delta}}-\sum_{j=m+1}^{N} \frac{\partial \xi^{i}}{\partial u^{\alpha}} \frac{\partial f^{i}}{\partial u^{\delta}},
$$

* Bianchi, loc. cit., p. 360 . 
and hence

$$
c_{\alpha \beta}^{(2)}=\sum_{i=0}^{m} \frac{\partial \xi^{i}}{\partial u^{\alpha}} \frac{\partial \xi^{i}}{\partial u^{\delta}}-\sum_{j=m+1}^{N} \frac{\partial \xi^{j}}{\partial u^{\alpha}} \frac{\partial \xi^{j}}{\partial u^{\delta}} .
$$

This is precisely the coefficient of the third fundamental form of the hypersurface for euclidean space* in which $m=n$.

If $\bar{V}_{n}$ is a hypersurface geodesically parallel to $V_{n}$ and if we denote by $\overline{\boldsymbol{g}}_{\alpha \beta}, \overline{\mathbf{\Omega}}_{\alpha \beta}, \bar{c}_{\alpha \beta}^{(2)}$ the coefficients of its three fundamental forms, we have from equations (5.3) that

$$
\begin{aligned}
& \bar{g}_{\alpha \beta}=g_{\alpha \beta}-2 \Omega_{\alpha \beta} c+\Omega_{\alpha \gamma} \Omega_{\beta}^{\gamma} c^{2}, \\
& \Omega_{\alpha \beta}=\Omega_{\alpha \beta}-\Omega_{\alpha \gamma} \Omega_{\beta}^{\gamma} c, \\
& \bar{c}_{\alpha \beta}^{(2)}=\Omega_{\alpha \gamma} \Omega_{\beta}^{\gamma},
\end{aligned}
$$

where $c$ is the constant distance from $\bar{V}_{n}$ to $V_{n}$. We observe

In a flat space, hypersurfaces geodesically parallel have the same third fundamental form.

The last two theorems of $\$ 12$ hold for flat spaces but as the proof for the special case is similar to the proof for the general case we shall give only the latter in the later section.

11. Let us next consider the case when $V_{n}$ is such that $R_{i j k l, m}=0$. This group of spaces contains as a subset the spaces of constant Riemann curvature; for if the curvature of. $V_{n+1}$ has the constant value of $K_{0}$, then $\dagger$

$$
R_{i j k l}=K_{0}\left(a_{i k} a_{j l}-a_{i l} a_{j k}\right),
$$

and from this it follows that $R_{i j k l, m}=0$. We can give a geometrical interpretation of the vanishing of $R_{i j k l, m}$ by means of the following theorems.

Let $\lambda^{i}(i=0,1,2, \cdots, n)$ be the components of an arbitrary contravariant vector, defining the congruence of curves whose differential equations are

$$
\frac{d x^{0}}{\lambda^{0}}=\frac{d x^{1}}{\lambda^{1}}=\cdots=\frac{d x^{n}}{\lambda^{n}}
$$

Further, let $\lambda_{1}{ }^{i}$ and $\lambda_{21}{ }^{i}$ be the contravariant components of two vectors arbitrary except that each forms a family of parallels along and with respect to the curves of the given congruence, in the sense of Levi-Civita.

* Bianchi, loc. cit., p. 474 ; cf. Eisenhart, loc. cit., p. 219, for the general flat space.

† Bianchi, loc. cit., pp. 75, 343. 
Then

A necessary and sufficient condition that the Riemannian curvature of (1.1) be constant along the curves of the given congruence and for any pair of directions of the type of $\lambda_{1 \mid}$ and $\lambda_{2 \mid}$ is that $R_{i j k l, m} \lambda^{m}$ vanish.

Before we prove this, let us observe that an immediate consequence of it is the following theorem:

If the curvature is constant along the curves of $n+1^{*}$ independent congruences in arbitrary pairs of directions which remain parallel, then $R_{i j k l, m}$ vanishes.

Let us return to the proof of the first theorem. The Riemann curvature $K$ in the directions $\lambda_{11}{ }^{i}$ and $\lambda_{21}{ }^{i}$ when $\lambda_{11}{ }^{i}$ and $\lambda_{21}{ }^{i}$ are orthogonal unit vectors, is given by $\dagger$

$$
K= \pm\left. R_{i j k i} \lambda_{1 \mid}^{i} \lambda_{2}\right|^{i} \lambda_{1}{ }^{k} \lambda_{21}{ }^{l}
$$

If each of the directions $\lambda_{1 \mid}$ and $\lambda_{2 \mid}$ forms a system of parallels, in the sense of Levi-Civita, aiong the curves of a congruence defined by a vector $\lambda^{i}$ we must have that $\ddagger$

$$
\left.\lambda_{\epsilon}\right|^{i} \cdot{ } \lambda^{i}=0 \quad(\epsilon=1,2 ; i=0,1, \cdots n)
$$

where $\lambda_{\epsilon \mid}{ }^{i}, j$ is the covariant derivative of $\lambda_{\epsilon}{ }^{i}$ with respect to $x^{i}$ and the fundamental form (1.1). Differentiate (11.2) covariantly with respect to $x^{m}$, multiply by $\lambda^{m}$ and sum for $m$; because of (11.3) we obtain

$$
\frac{\partial K}{\partial x^{m}} \lambda^{m}=R_{i j k l, m} \lambda_{1}{ }^{i} \lambda_{2}{ }^{i} \lambda_{1}{ }^{k} \lambda_{2 \mid}{ }^{l} \lambda^{m} .
$$

If $K$ is constant along the curves defined by $\lambda^{i},\left(\partial K^{i} / \partial x^{m}\right) \lambda^{m}$ must vanish and since $\lambda_{11}$ and $\lambda_{21}$ are arbitrary, we have from (11.4) and (1.12) that

$$
\left(R_{i j k l, m}+R_{k j i l, m}\right) \lambda^{m}=0 \quad(i, j, k, l=0,1,2, \cdots, n) .
$$

Since this holds for all values of $i, j, k$ and $l$, we have also

$$
-\left(R_{i k l i, m}+R_{l k i j, m}\right) \lambda^{m}=0 .
$$

Furthermore, from (1.11) we have

$$
\left(R_{i j k l, m}+R_{i k l i, m}+R_{i l j k, m}\right) \lambda^{m}=0 .
$$

* Note $n+1$ is the dimensionality of the space.

$\dagger$ Bianchi in deriving this formula does not assume that $\left.\lambda_{1}\right|^{i}$ and $\left.\lambda_{2}\right|^{i}$ are unit orthogonal vectors, so his value of $K$, loc. cit., p. 343, differs somewhat from (11.2). In what we do here, it is no restriction to make this hypothesis.

¥ Levi-Civita on Parallelism, Rendiconti del Circolo Matematico di Palermo, vol. 42 (1917), or Bianchi, Rendiconto della Reale Accademia delle Scienze Fisichee Matematiche di Napoli, ser. 3, vol. 27 (1922). 
Add these three equations, and we find

$$
R_{i j k l, m} \lambda^{m}=0 \text {. }
$$

Conversely, if (11.5) hold, from (11.4), $\left(\sigma K / \partial x^{m}\right) \lambda^{m}$ vanishes, and $K$ is constant along the curves defined by $\lambda^{i}$ in directions parallel with respect to these curves. This proves the theorem.

For a space satisfying the condition of the second theorem* the sequence of tensors (8.2) becomes the following:

$$
\begin{aligned}
& c_{\alpha \beta}^{(2)}=R_{0 \alpha \beta 0}+\Omega_{\alpha \gamma} \Omega_{\beta}^{\lambda}, \\
& c_{\alpha \beta}^{(3)}=-\frac{4}{3 !}\left(R_{0 \alpha \delta 0} \Omega_{\beta}^{\delta}+R_{0 \beta \delta 0} \Omega_{\alpha}^{\delta}\right),
\end{aligned}
$$

$$
\begin{aligned}
& c_{\alpha \beta}^{(2 m)}=\frac{2^{2 m-1}}{(2 m) !} R_{0 \lambda_{2} 0}^{\lambda_{1}} R_{0 \lambda_{3} 0}^{\lambda_{2}} \cdots R_{0 \lambda_{m-1} 0}^{\lambda_{m-2}}\left(R_{0 \alpha 0}^{\lambda_{m-1}} R_{0 \lambda_{1} \beta 0}+\Omega_{\alpha}^{\lambda_{m}-1} \Omega_{\beta}^{\mu} R_{0 \lambda_{1} \mu 0}\right), \\
& c_{\alpha \beta}^{(2 m+1)}=\frac{-2^{2 m}}{(2 m+1) !} R_{0 \lambda_{2} 0}^{\lambda_{1}} R_{0 \lambda_{3} 0}^{\lambda_{2}} \cdots R_{0 \lambda_{m-1} 0}^{\lambda_{m-2}} R_{0 \lambda_{m} 0}^{\lambda_{m-1}}\left(R_{0 \alpha \lambda_{1} 0} \Omega_{\beta}^{\lambda_{m}}+R_{0 \beta \lambda_{1} 0} \Omega_{\alpha}^{\lambda_{m}}\right) \\
& \quad(m=2,3, \cdots) .
\end{aligned}
$$

For the more restricted case of spaces of constant curvature equations (11.1) hold and in the coördinate system of (4.9) we have

$$
R_{0 \alpha \beta 0}=-e K_{0} g_{\alpha \beta}, \quad R_{0 \beta 0}^{\alpha}=-e K_{0} \delta_{\beta}^{\alpha} .
$$

Hence (11.6) become in this case

$$
\begin{aligned}
c_{\alpha \beta}^{(2)} & =-e K_{0} g_{\alpha \beta}+\Omega_{\alpha \gamma} \Omega_{\beta}^{\gamma}, \\
c_{\alpha \beta}^{(2 m)} & =(-e)^{m-1} \frac{2^{2 m-1}}{(2 m) !} K_{0}^{m-1} c_{\alpha \beta}^{(2)}, \\
c_{\alpha \beta}^{(2 m+1)} & =-(-e)^{m} \frac{2^{2 m+1}}{2 m !} K_{0}^{m} \Omega_{\alpha \beta} \quad(m=1,2, \cdots) .
\end{aligned}
$$

The summation of (5.3) can be effected quite easily; we obtain

$$
\begin{aligned}
c_{\alpha \beta}= & g_{\alpha \beta}-\frac{1}{\sqrt{e K_{0}}} \Omega_{\alpha \beta} \sin \left(2 \sqrt{e K_{0}} u^{0}\right) \\
& +\frac{1}{\eta_{e K_{0}}}\left\{1-\cos \left(2 \sqrt{e K_{0}} u^{0}\right)\right\} c_{\alpha \beta}^{(2)} \dagger
\end{aligned}
$$

* That is, if $R_{i j k l, m}=0$.

$\dagger$ If $e K_{0}$ has a negative value, (11.8) must be replaced by a similar formula with hyperbolic functions instead of trigonometric. 
Hence we may state the following theorem:

In a space of constant curvature, $K_{0}$, the fundamental tensor of a hypersurface geodesically parallel to and at a distance $u^{0}$ from an arbitrary hypersurface is given by (11.8).

By differentiation of (11.8) we obtain that for hypersurfaces geodesically parallel

$$
\begin{aligned}
\bar{\Omega}_{\alpha \beta} & =\Omega_{\alpha \beta} \cos \left(2 \sqrt{e K_{0}} u^{0}\right)-c_{\alpha \beta}^{(2)} \frac{1}{\sqrt{e K_{0}}} \sin \left(2 \sqrt{e K_{0}} u^{0}\right), \\
\bar{c}_{\alpha \beta}^{(2)} & =\Omega_{\alpha \beta} 2 \sqrt{e K_{0}} \sin \left(2 \sqrt{e K_{0}} u^{0}\right)+c_{\alpha \beta}^{(2)} \cos \left(2 \sqrt{e K_{0}} u^{0}\right) .
\end{aligned}
$$

We shall return to the consideration of these equations at the end of the next section.

12. Let us finally consider a special type of hypersurfaces, which, in one sense, are analogous in the general space to spheres and planes in euclidean space. Bianchi has shown* that the curvature, $1 / R$, of the geodesics of $V$ is given by

$$
\frac{1}{R}=\frac{\Omega_{\alpha \beta} d u^{\alpha} d u^{\beta}}{g_{\alpha \beta} d u^{\alpha} d u^{\beta}} .
$$

The maxima and minima of $1 / R$ are given by the determinantal equation

$$
\left|\Omega_{\alpha \beta}-\frac{1}{R} g_{\alpha \beta}\right|=0,
$$

and the corresponding directions, called the principal directions, are solutions of

$$
\left(\Omega_{\alpha \beta}-\frac{1}{R} g_{\alpha \beta}\right) \lambda^{\beta}=0 \quad(\alpha=1,2, \cdots, n) .
$$

There are $n$ sets of principal directions corresponding to the $n$ roots of (12.2); the $n$ congruences of curves whose directions at every point coincide with the principal directions are the lines of curvature; $1 / R$ is called the normal curvature of the hypersurface.

It follows immediately that a necessary and sufficient condition that the lines of curvature of a hypersurface be completely indeterminate is that $\Omega_{\alpha \beta}=(1 / R) g_{\alpha \beta}$ for all $\alpha$ and $\beta$. These hypersurfaces are, in one sense, generalizations of spheres and planes; more particularly if $1 / R=0$ we have the type of hypersurface mentioned in $\S 9$. We shall prove the following theorem:

- Bianchi, loc. cit., p. 366; Eisenhart, loc. cit., p. 151. 
In a space of constant Riemann curvature, the hypersurfaces whose lines of curvature are completely indeterminate have constant normal curvature and constant Riemann curvature; conversely if a hypersurface of space of constant Riemann curvature has constant Riemann curvature, then in the enveloping space it has constant normal curvature and completely indeterminate lines of curvature. The Riemann curvature of such a hypersurface is equal to the sum (or difference) of the square of its normal curvature and the Riemann curvature of the enveloping space.*

The proof is an immediate consequence of the equations of Gauss $\dagger$ connecting the first and second fundamental forms of a hypersurface. For spaces of constant curvature, $K_{0}$, these equations are

$$
e\left(\Omega_{\alpha \gamma} \Omega_{\beta \delta}-\Omega_{\alpha \delta} \Omega_{\beta \gamma}\right)=\bar{R}_{\alpha \beta \gamma \delta}-K_{0}\left(g_{\alpha \gamma} g_{\beta \delta}-g_{\alpha \delta} g_{\beta \gamma}\right),
$$

where $\bar{R}_{\alpha \beta \gamma \delta}$ is the Riemann tensor formed with respect to the quadric form (4.3) and $e$ is defined by (4.9). If the hypersurface has indeterminate lines of curvature, $\Omega_{\alpha \beta}=(1 / R) g_{\alpha \beta}$ and consequently (12.4) become

$$
\bar{R}_{\alpha \beta \gamma \delta}=\left(\frac{e}{R^{2}}+K_{0}\right)\left(g_{\alpha \gamma} g_{\beta \delta}-g_{\alpha \delta} g_{\beta \gamma}\right) .
$$

From the theorem of Schur $\ddagger$ it follows that $e / R^{2}+K_{0}$ is constant, and from (11.1) it is the value of the Riemann curvature of $V_{n}$. Conversely, if (12.5) are satisfied, from (12.4) it follows that every two-row determinant from the square matrix $\left\|\Omega_{\alpha \beta}\right\|$ is equal to the corresponding two-row determinant from $\left\|g_{\alpha \beta} / R\right\|$. Furthermore, if $1 / R \neq 0$ there is a three-row determinant from the latter matrix different from zero, since the determinant $\left|g_{\alpha \beta}\right| \neq 0$. It then follows from a theorem due to Killing $\S$ that the elements of the two matrices differ at most in sign; that is

$$
\Omega_{\alpha \beta}= \pm \frac{1}{R} g_{\alpha \beta} \quad(\alpha, \beta=1,2, \cdots, n) .
$$

If $1 / R=0$, from (12.1) we have that $\Omega_{\alpha \beta}=0$ and (12.6) hold in this case too. This proves the theorem.

We observe that $e$ is necessarily positive if the form (1.1) or its equivalent (4.9) is positive definite; hence we have the following immediate corollary:

In spaces of more than three dimensions of constant Riemann curvature, $K_{0}$, whose fundamental form is positive definite, there are no real hypersurfaces of constant curvature $K<K_{0}$.

* The converse theorem holds only for $n>2$; the direct theorem however is always true.

† Bianchi, loc. cit., p. 362; Eisenhart, loc. cit., p. 150.

$\ddagger$ Mathematische Annalen, vol. 27, p. 563 .

$\S$ Nicht-Euklidische Raumformen in Analytischer Behandlung, Leipzig, Teubner, 1885, pp. 236-237 
As a special case we have the well known theorem that in euclidean 4-space there are no 3 -spaces of constant negative curvature.

If equations (12.6) hold, we find from (11.8), by virtue of the first of (11.7), that $c_{\alpha \beta}$ is proportional to $g_{\alpha \beta}$ and hence the map between $V_{n}$ and $\bar{V}_{n}$ established by the geodesics of $V_{n+1}$ normal to $V_{n}$ is conformal. Conversely if a hypersurface is mapped conformally on all the hypersurfaces geodesically parallel to it, from (11.8) we obtain that $\Omega_{\alpha \beta}$ and $c_{\alpha \beta}^{(2)}$ are proportional to $g_{\alpha \beta}$. We observe further from (11.9) that, if $\Omega_{\alpha \beta}=(1 / R) g_{\alpha \beta}$, we have that $\bar{\Omega}_{\alpha \beta}=(1 / \bar{R}) c_{\alpha \beta}$. Hence we have the following theorems:

If, in a space of constant curvature, a hypersurface is mapped conformally on the hypersurfaces geodesically parallel to it, the map being established by the normal geodesics, then these hypersurfaces have completely indeterminate lines of curvature.

The hypersurfaces geodesically parallel to a hypersurjace with indeterminate lines of curvature in a space of constant Riemann curvature are themselves hypersurfaces with indeterminate lines of curvature.

Because of the first theorem in this section we may restate the preceding:

In a space of constant Riemann curvature, a hypersurface geodesically parallel to a hypersurface of constant Riemann curvature must also be of constant Riemann curvature.

In a flat space hypersurfaces with indeterminate lines of curvature must be hyperplanes or hyperspheres, and so the last two theorems are trivial.

From (12.3) we readily obtain that

$$
\Omega_{\alpha \beta} \lambda_{\left.h\right|^{\alpha}} \lambda_{k \mid}{ }^{\beta}=0, \quad g_{\alpha \beta} \lambda_{h \mid}{ }^{\alpha} \lambda_{k \mid}{ }^{\beta}=0 \quad(h, k=1,2, \cdots, n ; h \neq k),
$$

and from (11.8) and (11.9) by virtue of (12.3) and (12.6) it follows that

$$
\begin{aligned}
& c_{\alpha \beta} \lambda_{\left.h\right|^{\alpha}} \lambda_{\left.k\right|^{\beta}}=0, \\
& \bar{\Omega}_{\alpha \beta} \lambda_{\left.h\right|^{\alpha}} \lambda_{k \mid}{ }^{\beta}=0 \quad(h, k=1,2, \cdots, n ; h \neq k) .
\end{aligned}
$$

Equations (12.7) are also sufficient conditions that the congruences $\lambda_{h}{ }^{\alpha}$ $(h=1,2, \cdots, n)$ be lines of curvature, for if in (12.7) we let $h$ be fixed, and $k$ take on the values $1,2, \cdots, n, \neq h$ it follows that the vector $\Omega_{\alpha \beta} \lambda_{h \mid}{ }^{\alpha}$ is normal to the $n-1$ vectors $\lambda_{k}$; hence $\Omega_{\alpha \beta} \lambda_{h_{1}}{ }^{\alpha}=\rho \lambda_{h_{1} \beta}$ or $\lambda_{h_{1}}$ is a line of curvature. Consequently we have the following theorem, which holds also in euclidean space :

In a space of constant curvature the lines of curvature of geodesically parallel hypersurfaces correspond.

Princeton UnIVERSITY, Princeton, N. J. 